\title{
AiMT
}

Advances in Military Technology

Vol. 15, No. 2, 2020, pp. 405-423

ISSN 1802-2308, eISSN 2533-4123

DOI 10.3849/aimt.01379

\section{Effect of Some Disturbance Factors on the Motion Stability of Unguided Rockets}

\author{
H. Ban $\mathrm{Le}^{1}$ and P. Konečný ${ }^{2 *}$ \\ ${ }^{1}$ Le Quy Don Technical University, Hanoi, Vietnam \\ ${ }^{2}$ University of Defence in Brno, Czech Republic
}

The manuscript was received on 21 January 2020 and was accepted after revision for publication as research paper on 2 November 2020.

\begin{abstract}
:
A mathematical model of unguided-rockets' motion in the air taking into account the deflection of the thrust and the position deviation of the center of gravity has been established. The mathematical model has been solved for a specific unguided-rocket slow spin to predict its standard trajectory and deviation trajectories; calculated results agree well with the corresponding data in the firing table. The effect of the disturbance factors on the motion stability is shown through the deviation of the graph of the angle of attack; sideslip angle and the trajectory falling points compared to the corresponding values of the standard trajectory, which does not contain deviations.
\end{abstract}

\section{Keywords:}

deflection of the thrust, deviation of the center of gravity, motion stability, unguided rocket

\section{Introduction}

In addition to creating the rolling moment, a rocket motor thrust is often designed so that its direction coincides with the rocket's symmetry axis. Besides, the rocket's center of gravity is usually designed to lie on the symmetry axis. However, due to manufacturing and assembly errors the thrust and the rocket's center of gravity deviate from their design positions. These deviations are preliminarily shown in Fig. 1, where $\lambda_{T}$ is the deflection angle between the thrust and the rocket's symmetry axis, $\lambda_{T}$ is called the thrust deflection; $e_{\mathrm{m}}$ is the deviation of the rocket's center of gravity, $O$ from its design position, $O$ ' (which is on the geometric axis).

\footnotetext{
* Corresponding author: Department of Weapons and Ammunition, University of Defence in Brno, Kounicova 156/65, CZ-662 10 Brno, Czech Republic. Phone: +420 973442 037, E-mail: pavel.konecny@unob.cz,ORCID 0000-0003-0703-2315
} 


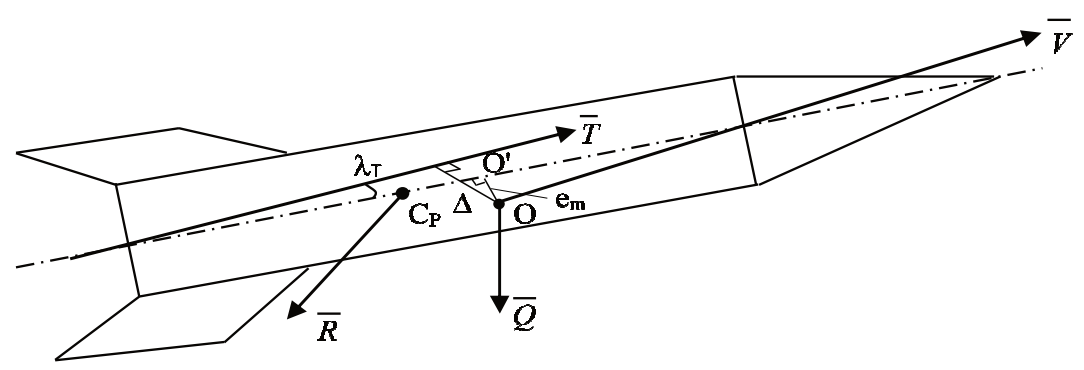

Fig. 1 Combination of thrust deflection and deviation of the center of gravity

The deviation of the center of gravity causes a change in the inertial axes and the moments of inertia of the rocket. In addition, this deviation combined with the thrust deflection creates a thrust eccentricity, $\Delta$ which forms unwanted moments. Each deviation acts as a disturbance factor that affects the rocket's motion on the flight.

The mentioned disturbances distribute in all different directions with respect to the rocket's body, each disturbance factor mentioned has a distinct effect on the stability of rocket motion in the air. When these disturbance factors are combined, one disturbance factor can increase or decrease the effects of the remaining factors on the stability of rocket motion. So, determining the combinative effect of these disturbance factors is necessary, in order to find a limit for them, ensuring the accuracy of the firing process.

According to the general concepts of stability and technical requirements of weapons, a rocket is considered stable if its oscillation on the flight is stable and its trajectory falling point within an allowable limit $[1,2]$. Rocket oscillation is stable if the amplitudes of the angle of attack and sideslip angle decrease to zero. The allowable limit of falling points is taken as the allowable falling point distribution area in the firing table, ensuring firing accuracy.

The article establishes a mathematical model for unguided-rocket motion in the air taking into account the mentioned disturbance factors, then investigating their effects on the motion stability of a fin-stabilized rocket, spinning slowly. The criteria for evaluating motion stability are the laws of change of the attack angle; sideslip angle and the dispersion of the trajectory falling points.

\section{Mathematical Model of Unguided Rocket Motion in the Air Taking into Account the Thrust Deflection and the Deviation of the Center of Gravity}

\subsection{Coordinate Systems}

For investigating the rocket motion in the air, the article uses the following coordinate systems $[3,4]$ :

- normal earth coordinate system, $O_{\mathrm{g}} X_{\mathrm{g}} Y_{\mathrm{g}} Z_{\mathrm{g}}$ is a fixed coordinate system on the ground with its origin, $O_{\mathrm{g}}$ coinciding with the rocket's center of gravity at the moment the rocket leaves the launcher; the axis, $O_{\mathrm{g}} X_{\mathrm{g}}$ is the intersection of the firing plane (the vertical plane containing the rocket axis when starting to launch) with the horizontal plane across the origin. It is positive in the firing direction; the axis, $O_{\mathrm{g}} Z_{\mathrm{g}}$, is perpendicular to $O_{\mathrm{g}} X_{\mathrm{g}}$, and downward; the axis, $O_{\mathrm{g}} Y_{\mathrm{g}}$, is determined according to right rotation rule, 
- earth coordinate system attached to rocket, $O x_{\mathrm{g}} y_{\mathrm{g}} z_{\mathrm{g}}$ has the origin in the rocket's center of gravity, $O$; its axes are always parallel with the axes of the normal earth coordinate system. $\left(O x_{\mathrm{g}}\left\|O_{\mathrm{g}} X_{\mathrm{g}} ; O y_{\mathrm{g}}\right\| O_{\mathrm{g}} Y_{\mathrm{g}} ; O z_{\mathrm{g}} \| O_{\mathrm{g}} Z_{\mathrm{g}}\right)$,

- aerodynamic coordinate system $O X_{\mathrm{a}} Y_{\mathrm{a}} Z_{\mathrm{a}}$ can be identical to the flight path coordinate system for an axially symmetrical rocket. Aerodynamic roll angle is not necessary in this case. The coordinate system is attached to the velocity vector of the rocket's center of gravity, $O X_{\mathrm{a}}$ coincides with vector $V ; O Z_{\mathrm{a}}$ is perpendicular to $O X$ in a vertical plane passing through $V$ and downward; $O Y_{\mathrm{a}}$ is perpendicular to the plane $O X_{\mathrm{a}} Z_{\mathrm{a}}$ in a right rotation rule. The position angles between the aerodynamic coordinate system and normal earth coordinates system are: $\chi_{\mathrm{a}}-$ aerodynamic azimuth, $\gamma_{a}-$ aerodynamic pitch,

- body coordinate system, $O X Y Z$ determines the position of the rocket axes. Its origin coincides with the rocket's center of gravity; $O X$ is parallel to the rocket's symmetry axis (In case the center of gravity coincides with the geometric center, then $O X$ coincides with the rocket's symmetry axis); $O Z$ is in the vertical plane and downward; $O Y$ is determined according to right rotation rule. The rocket's spin motion is determined by the spin angle, $v$ around the axis, $O X$. The position angles between the body coordinate system and the velocity coordinate system: $\alpha$-angle of attack, $\beta$ - sideslip angle.

Geometric relationship between coordinate systems $O x_{\mathrm{g}} y_{\mathrm{g}} z_{\mathrm{g}} ; O X_{\mathrm{a}} Y_{\mathrm{a}} Z_{\mathrm{a}}$ and $O X Y Z$ is shown in Fig. 2.

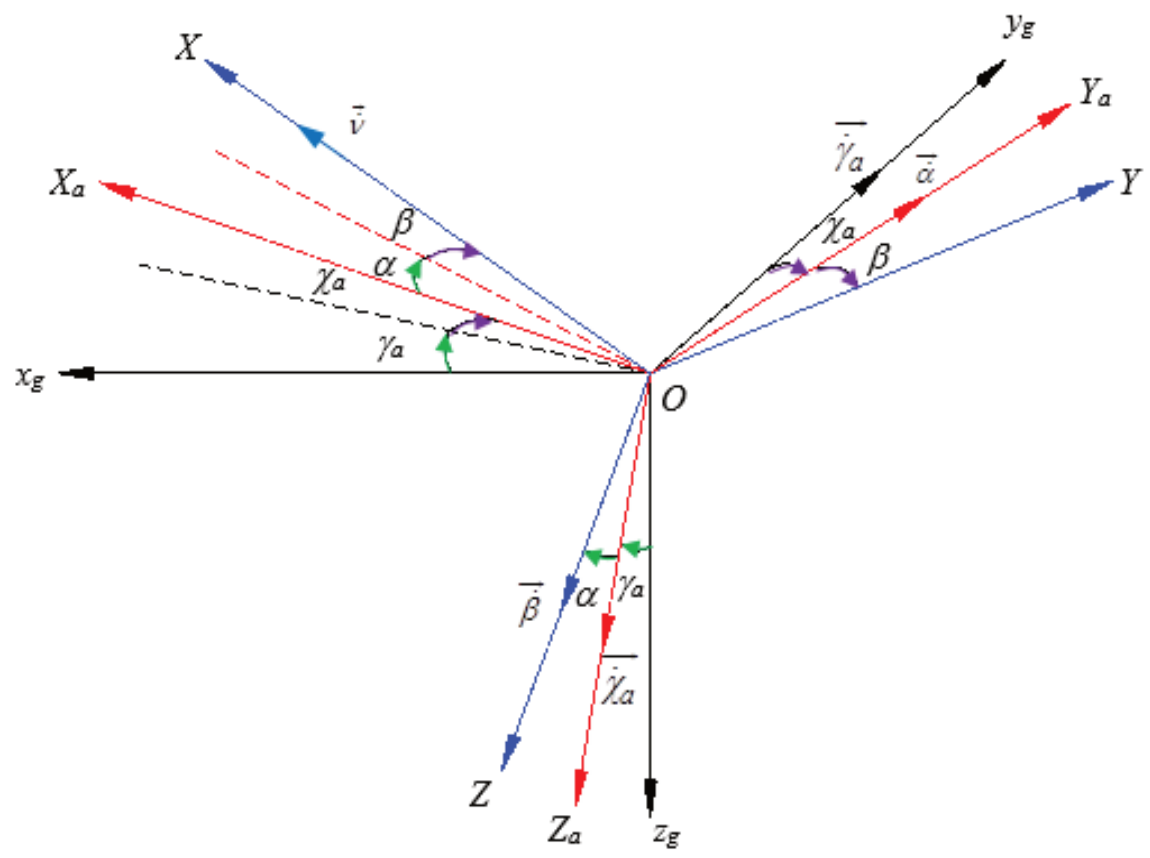

Fig. 2 Geometric relationship between coordinate systems 
Transformation matrices between the coordinate systems:

$$
\begin{aligned}
& {\left[\begin{array}{l}
x_{\mathrm{g}} \\
y_{\mathrm{g}} \\
z_{\mathrm{g}}
\end{array}\right]=\left[\begin{array}{ccc}
\cos \gamma_{\mathrm{a}} \cos \chi_{\mathrm{a}} & -\cos \gamma_{\mathrm{a}} \sin \chi_{\mathrm{a}} & \sin \gamma_{\mathrm{a}} \\
\sin \chi_{\mathrm{a}} & \cos \chi_{\mathrm{a}} & 0 \\
-\sin \gamma_{\mathrm{a}} \cos \chi_{\mathrm{a}} & \sin \gamma_{\mathrm{a}} \sin \chi_{\mathrm{a}} & \cos \gamma_{\mathrm{a}}
\end{array}\right]\left[\begin{array}{l}
x_{\mathrm{a}} \\
y_{\mathrm{a}} \\
z_{\mathrm{a}}
\end{array}\right]} \\
& {\left[\begin{array}{l}
x_{\mathrm{a}} \\
y_{\mathrm{a}} \\
z_{\mathrm{a}}
\end{array}\right]=\left[\begin{array}{ccc}
\cos \alpha \cos \beta & -\cos \alpha \sin \beta & \sin \alpha \\
\sin \beta & \cos \beta & 0 \\
-\sin \alpha \cos \beta & \sin \alpha \sin \beta & \cos \alpha
\end{array}\right]\left[\begin{array}{l}
x \\
y \\
z
\end{array}\right]}
\end{aligned}
$$

\subsection{Assumptions}

In the mathematical model, the article uses some assumptions as follows:

- the center of gravity has a position deviation in the direction perpendicular to the rocket axis $O X$,

- when the position of the center of gravity changes, principal axes of inertia will remain parallel to their design positions (which coincide with the rocket's geometric axes),

- the asymmetry in mass distribution is the same along the rocket's symmetry axis. When the rocket motor works, the rocket's center of gravity moves in the direction parallel to the rocket axis $O X$,

- rocket motor operates stably and the thrust does not change its direction during operation,

- the effects of wind, initial disturbance, Earth curvature, Magnus force and Coriolis force are ignored.

\subsection{Combination of the Thrust Deflection and the Deviation of the Rocket's Center of Gravity}

Suppose the thrust deflection and the deviation of the rocket's center of gravity at the initial time are represented through geometric parameters as shown in Fig. 3.

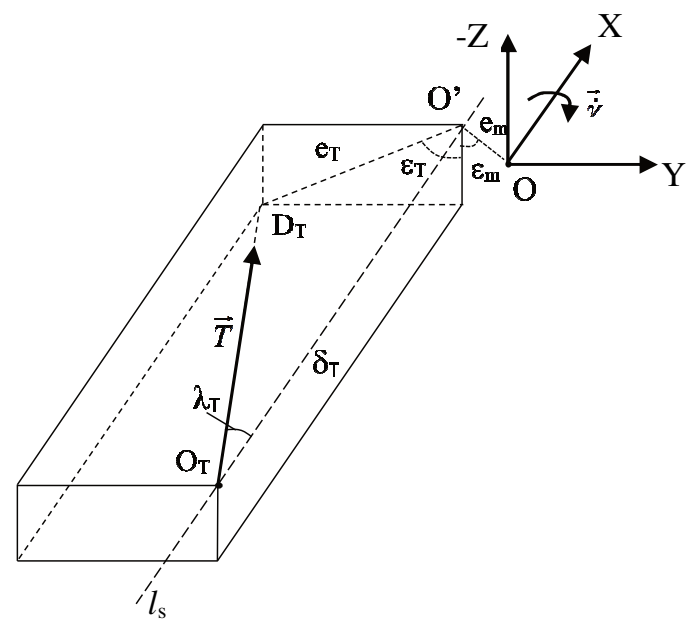

Fig. 3 Geometric parameters of thrust deflection and deviation of the center of gravity 
where

- $l_{\mathrm{s}}$ - the rocket's geometrical symmetry axis,

- $O^{\prime}$ - the design position of the center of gravity,

- $O_{T}$ - the distributed center of the motor nozzles' critical sections,

- $\quad \delta_{\mathrm{T}}-$ the distance between $O^{\prime}$ and $O_{\mathrm{T}}$,

- $T$ - the total thrust generated by the rocket motor system,

- $\lambda_{\mathrm{T}} ; \mathcal{E}_{\mathrm{T}}-$ the position deviation parameters of thrust with respect to its design position,

- $e_{\mathrm{m}} ; \varepsilon_{\mathrm{m}}-$ the position deviation parameters of the center of gravity, $O$ with respect to its design position, $O^{\prime}$.

After a period of time $t$, the rocket rotates around the axis, $O X$ an angle, $v=\int_{0}^{t} \dot{v} d t$.

With a convention that the positive direction of angles $\varepsilon_{\mathrm{T}}$ and $\varepsilon_{\mathrm{m}}$ is anticlockwise when viewing from the top of vector $O X$, according to Fig. 3, the position of the point, $O^{\prime}$ and the thrust, $T$ in the Body coordinate system are as follows:

$$
\begin{aligned}
& {\left[\begin{array}{l}
x_{O^{\prime}} \\
y_{O^{\prime}} \\
z_{O^{\prime}}
\end{array}\right]=\left[\begin{array}{c}
0 \\
e_{\mathrm{m}} \sin \left(\varepsilon_{\mathrm{m}}+v\right) \\
-e_{\mathrm{m}} \cos \left(\varepsilon_{\mathrm{m}}+v\right)
\end{array}\right]} \\
& {\left[\begin{array}{l}
T_{x} \\
T_{y} \\
T_{z}
\end{array}\right]=\left[\begin{array}{c}
T \cos \lambda_{\mathrm{T}} \\
-T \sin \lambda_{\mathrm{T}} \sin \left(\varepsilon_{\mathrm{T}}+v\right) \\
T \sin \lambda_{\mathrm{T}} \cos \left(\varepsilon_{\mathrm{T}}+v\right)
\end{array}\right]}
\end{aligned}
$$

From Eqs (1) and (3), thrust components on the velocity coordinate system are:

$$
\left[\begin{array}{l}
T_{x_{\mathrm{a}}} \\
T_{y_{\mathrm{a}}} \\
T_{z_{\mathrm{a}}}
\end{array}\right]=\left[\begin{array}{c}
T \cos \lambda_{\mathrm{T}} \cos \alpha \cos \beta+T \sin \lambda_{\mathrm{T}}\left[\cos \left(\varepsilon_{\mathrm{T}}+v\right) \sin \alpha+\sin \left(\varepsilon_{\mathrm{T}}+v\right) \cos \alpha \sin \beta\right] \\
T \cos \lambda_{\mathrm{T}} \sin \beta-T \sin \lambda_{\mathrm{T}} \sin \left(\varepsilon_{\mathrm{T}}+v\right) \cos \beta \\
-T \cos \lambda_{\mathrm{T}} \sin \alpha \cos \beta+T \sin \lambda_{\mathrm{T}}\left[\cos \left(\varepsilon_{\mathrm{T}}+v\right) \cos \alpha-\sin \left(\varepsilon_{\mathrm{T}}+v\right) \sin \alpha \sin \beta\right]
\end{array}\right]
$$

From Eqs (2) and (3), moment components (generated by thrust $T$ ) on Body coordinate system:

$$
\begin{aligned}
& {\left[\begin{array}{l}
M_{x} \\
M_{y} \\
M_{z}
\end{array}\right]=\left[\begin{array}{c}
-T_{y} \cdot z_{O^{\prime}}+T_{z} \cdot y_{O^{\prime}} \\
-T_{x} \cdot z_{O^{\prime}}+T_{z} \cdot \delta_{\mathrm{T}} \\
-T_{x} \cdot y_{O^{\prime}}+T_{y} \cdot \delta_{\mathrm{T}}
\end{array}\right]} \\
& {\left[\begin{array}{c}
M_{x} \\
M_{y} \\
M_{z}
\end{array}\right]=\left[\begin{array}{c}
-T \sin \lambda_{\mathrm{T}} \cdot e_{\mathrm{m}}\left[\sin \left(\varepsilon_{\mathrm{T}}+v\right) \cos \left(\varepsilon_{\mathrm{m}}+v\right)-\cos \left(\varepsilon_{\mathrm{T}}+v\right) \sin \left(\varepsilon_{\mathrm{m}}+v\right)\right] \\
-T \cos \lambda_{\mathrm{T}} \cdot e_{\mathrm{m}} \cos \left(\varepsilon_{\mathrm{m}}+v\right)+T \sin \lambda_{\mathrm{T}} \cos \left(\varepsilon_{\mathrm{T}}+v\right) \cdot \delta_{\mathrm{T}} \\
-T \cos \lambda_{\mathrm{T}} \cdot e_{\mathrm{m}} \sin \left(\varepsilon_{\mathrm{m}}+v\right)+T \sin \lambda_{\mathrm{T}} \sin \left(\varepsilon_{\mathrm{T}}+v\right) \cdot \delta_{\mathrm{T}}
\end{array}\right]}
\end{aligned}
$$

\subsection{Mathematical Model}

\section{Translational motion equation}

Rockets' translational motion is represented in Flight-path axis system [3], as follows: 


$$
\begin{aligned}
m \cdot \dot{V} & =T_{x_{\mathrm{a}}}-D-m \cdot g \sin \gamma_{\mathrm{a}} \\
m \cdot V \cdot \dot{\gamma}_{\mathrm{a}} & =-T_{z_{\mathrm{a}}}+L-m \cdot g \cos \gamma_{\mathrm{a}} \\
m \cdot V \cos \gamma_{\mathrm{a}} \cdot \dot{\chi}_{\mathrm{a}} & =T_{y_{\mathrm{a}}}+Y_{\mathrm{a}}
\end{aligned}
$$

where

- $m$ - the rocket's mass at time t,

- $V$ - the velocity of rocket's center of gravity,

- $D, L, Y_{\mathrm{a}}$ - the drag; lift and lateral force, respectively,

- $g$ - the gravity acceleration, it changes with altitude of the rocket.

$$
g=g_{0} \frac{R^{2}}{\left(R-Z_{\mathrm{g}}\right)^{2}}
$$

$R$ - the radius of the Earth, $g_{0}$ - gravity acceleration at altitude 0 .

Eq. (6) is rewritten as:

$$
\begin{aligned}
& \dot{V}=\frac{1}{m}\left(T_{x_{a}}-D-m \cdot g \sin \gamma_{\mathrm{a}}\right) \\
& \dot{\gamma}_{\mathrm{a}}=\frac{1}{m \cdot V}\left(-T_{z_{a}}+L-m \cdot g \cos \gamma_{\mathrm{a}}\right) \\
& \dot{\chi}_{\mathrm{a}}=\frac{1}{m \cdot V \cos \gamma_{\mathrm{a}}}\left(T_{y_{\mathrm{a}}}+Y_{\mathrm{a}}\right)
\end{aligned}
$$

\section{Rotational motion equation}

Rocket's rotational motion is represented in the Body coordinate system [3, 4] as follows:

$$
\left(\frac{\partial \boldsymbol{H}}{\partial t}+\boldsymbol{\Omega} \times \boldsymbol{H}\right)=\sum \overline{\boldsymbol{M}}
$$

where

- $\boldsymbol{H}$ - the rocket's angular momentum in Body coordinate system. If the rocket's principal moments of inertia $\boldsymbol{I}$ and angular velocity with respect to the Body coordinate system are $I_{x}, I_{y}, I_{z}$ and $\omega=\left(\omega_{x}, \omega_{y}, \omega_{z}\right)$, respectively, then:

$$
\boldsymbol{H}=\left(I_{x} \cdot \omega_{x} ; I_{y} \cdot \omega_{y} ; I_{z} \cdot \omega_{z}\right)
$$

- $\boldsymbol{\Omega}$ - the angular velocity of Body coordinate system: $\boldsymbol{\Omega}=\left(\omega_{x}-\dot{v} ; \omega_{y} ; \omega_{z}\right)$,

- $\overline{\boldsymbol{M}}$ - the resultant moment with respect to the center of gravity. Eq. (9) is rewritten as:

$$
\begin{aligned}
& I_{x} \cdot \dot{\omega}_{x}=M_{x}+L_{R} \\
& I_{y} \cdot \dot{\omega}_{y}+\left(I_{x}-I_{z}\right) \omega_{x} \cdot \omega_{z}+I_{z} \cdot \omega_{z} \cdot \dot{v}=M_{y}+M \\
& I_{z} \cdot \dot{\omega}_{z}+\left(I_{y}-I_{x}\right) \omega_{x} \cdot \omega_{y}-I_{y} \cdot \omega_{y} \cdot \dot{v}=M_{z}+N
\end{aligned}
$$

where $L_{R}, M, N$ - rolling moment; pitching moment and yawing moment, respectively.

Eq. (11) is rewritten as: 


$$
\begin{aligned}
& \dot{\omega}_{x}=\frac{1}{I_{x}}\left(M_{x}+L_{R}\right) \\
& \dot{\omega}_{y}=\frac{1}{I_{y}}\left[M_{y}+M-\left(I_{x}-I_{z}\right) \omega_{x} \cdot \omega_{z}-I_{z} \cdot \omega_{z} \cdot \dot{v}\right] \\
& \dot{\omega}_{z}=\frac{1}{I_{z}}\left[M_{z}+N-\left(I_{y}-I_{x}\right) \omega_{x} \cdot \omega_{y}+I_{y} \cdot \omega_{y} \cdot \dot{v}\right]
\end{aligned}
$$

\section{Kinematic relationships}

According to Fig. 2 and Eq. (1), kinematic relationships are represented as follows:

$$
\begin{aligned}
& \omega_{x}=\dot{v}+\dot{\alpha} \sin \beta+\dot{\gamma}_{\mathrm{a}}\left(\cos \alpha \cos \beta \sin \chi_{\mathrm{a}}+\sin \beta \cos \chi_{\mathrm{a}}\right)-\dot{\chi}_{\mathrm{a}} \sin \alpha \cos \beta \\
& \omega_{y}=\dot{\alpha} \cos \beta+\dot{\gamma}_{\mathrm{a}}\left(\cos \beta \cos \chi_{\mathrm{a}}-\cos \alpha \sin \beta \sin \chi_{\mathrm{a}}\right)+\dot{\chi}_{\mathrm{a}} \sin \alpha \sin \beta \\
& \omega_{z}=\dot{\beta}+\dot{\gamma}_{\mathrm{a}} \sin \alpha \sin \chi_{\mathrm{a}}+\dot{\chi}_{\mathrm{a}} \cos \alpha
\end{aligned}
$$

Eq. (13) is rewritten as:

$$
\begin{aligned}
& \dot{\alpha}=\frac{1}{\cos \beta}\left[\omega_{y}-\dot{\gamma}_{\mathrm{a}}\left(\cos \beta \cos \chi_{\mathrm{a}}-\cos \alpha \sin \beta \sin \chi_{\mathrm{a}}\right)-\dot{\chi}_{\mathrm{a}} \sin \alpha \sin \beta\right] \\
& \dot{\beta}=\omega_{z}-\dot{\gamma}_{\mathrm{a}} \sin \alpha \sin \chi_{\mathrm{a}}-\dot{\chi}_{\mathrm{a}} \cos \alpha \\
& \dot{v}=\omega_{x}-\dot{\alpha} \sin \beta-\dot{\gamma}_{\mathrm{a}}\left(\cos \alpha \cos \beta \sin \chi_{\mathrm{a}}+\sin \beta \cos \chi_{\mathrm{a}}\right)+\dot{\chi}_{\mathrm{a}} \sin \alpha \cos \beta
\end{aligned}
$$

The velocity components of the rocket's center of gravity in Normal earth coordinate system:

$$
\begin{aligned}
& \dot{X}_{\mathrm{g}}=V \cos \gamma_{\mathrm{a}} \cos \chi_{\mathrm{a}} \\
& \dot{Y}_{\mathrm{g}}=V \cos \gamma_{\mathrm{a}} \sin \chi_{\mathrm{a}} \\
& \dot{Z}_{\mathrm{g}}=-V \sin \gamma_{\mathrm{a}}
\end{aligned}
$$

\section{Mathematical model}

From Eqs (4); (5); (8); (12); (14) and (15), mathematical model of unguided-rocket motion in the air taking into account the thrust deflection and the deviation of the center of gravity is expressed as Eq. (16).

The system of equations (16) includes variables as $V ; \gamma_{\mathrm{a}} ; \chi_{\mathrm{a}} ; \alpha ; \beta ; v ; \omega_{x} ; \omega_{y} ; \omega_{z} ; X_{\mathrm{g}}$; $Y_{\mathrm{g}} ; Z_{\mathrm{g}}$. The system is often solved by numerical methods, the initial condition for solving the system is the motion parameters at the time the rocket leaves the launcher. Also, the change of mass, moments of inertia and aerodynamic functions need to be determined.

\section{Simulating Motion in the Air of a Fin-Stabilized Rocket}

The motion of a representative rocket of a type of unguided-rockets, fin-stabilized with a single-stage solid-propellant rocket motor, which is BM21-rocket that are simulated, as a basis for investigating the effects of the disturbance factors mentioned on the rocket motion. 
Eq. (16):

$$
\left.\begin{array}{rl}
\dot{V}= & \frac{1}{m}\left\{T \cos \lambda_{\mathrm{T}} \cos \alpha \cos \beta+T \sin \lambda_{\mathrm{T}}\left[\cos \left(\varepsilon_{\mathrm{T}}+v\right) \sin \alpha+\sin \left(\varepsilon_{\mathrm{T}}+v\right) \cos \alpha \sin \beta\right]\right. \\
& \left.-D-m \cdot g \sin \gamma_{\mathrm{a}}\right\} \\
\dot{\gamma}_{\mathrm{a}}= & \frac{1}{m \cdot V}\left\{T \cos \lambda_{\mathrm{T}} \sin \alpha \cos \beta-T \sin \lambda_{\mathrm{T}}\left[\cos \left(\varepsilon_{\mathrm{T}}+v\right) \cos \alpha-\sin \left(\varepsilon_{\mathrm{T}}+v\right) \sin \alpha \sin \beta\right]\right. \\
& \left.+L-m \cdot g \cos \gamma_{\mathrm{a}}\right\} \\
\dot{\chi}_{\mathrm{a}}= & \frac{1}{m \cdot V \cos \gamma_{\mathrm{a}}}\left(T \cos \lambda_{\mathrm{T}} \sin \beta-T \sin \lambda_{\mathrm{T}} \sin \left(\varepsilon_{\mathrm{T}}+v\right) \cos \beta+Y_{\mathrm{a}}\right) \\
\dot{\alpha}= & \frac{1}{\cos \beta}\left[\omega_{y}-\dot{\gamma}_{a}\left(\cos \beta \cos \chi_{\mathrm{a}}-\cos \alpha \sin \beta \sin \chi_{\mathrm{a}}\right)-\dot{\chi}_{\mathrm{a}} \sin \alpha \sin \beta\right] \\
\dot{\beta}_{=}= & \omega_{z}-\dot{\gamma}_{\mathrm{a}} \sin \alpha \sin \chi_{\mathrm{a}}-\dot{\chi}_{\mathrm{a}} \cos \alpha \\
\dot{v}= & \omega_{x}-\dot{\alpha} \sin \beta-\dot{\gamma}_{\mathrm{a}}\left(\cos \alpha \cos \beta \sin \chi_{\mathrm{a}}+\sin \beta \cos \chi_{\mathrm{a}}\right)+\dot{\chi}_{\mathrm{a}} \sin \alpha \cos \beta \\
\dot{\omega}_{x}= & \frac{1}{I_{x}}\left(-T \sin \lambda_{\mathrm{T}} \cdot e_{\mathrm{m}}\left[\sin \left(\varepsilon_{\mathrm{T}}+v\right) \cos \left(\varepsilon_{m}+v\right)-\cos \left(\varepsilon_{\mathrm{T}}+v\right) \sin \left(\varepsilon_{m}+v\right)\right]+L_{R}\right) \\
\dot{\omega}_{y}= & \frac{1}{I_{y}}\left[-T \cos \lambda_{\mathrm{T}} \cdot e_{\mathrm{m}} \cos \left(\varepsilon_{m}+v\right)+T \sin \lambda_{\mathrm{T}} \cos \left(\varepsilon_{\mathrm{T}}+v\right) \cdot \delta_{\mathrm{T}}-\left(I_{x}-I_{z}\right) \omega_{x} \cdot \omega_{z}-I_{z} \cdot \omega_{z} \cdot \dot{v}+M\right] \\
\dot{\omega}_{z}= & \frac{1}{I_{z}}\left[-T \cos \lambda_{\mathrm{T}} \cdot e_{m} \sin \left(\varepsilon_{\mathrm{m}}+v\right)+T \sin \lambda_{\mathrm{T}} \sin \left(\varepsilon_{\mathrm{T}}+v\right) \cdot \delta_{\mathrm{T}}-\left(I_{y}-I_{x}\right) \omega_{x} \cdot \omega_{y}+I_{y} \cdot \omega_{y} \cdot \dot{v}+N\right] \\
\dot{X}_{g}= & V \cos \gamma_{\mathrm{a}} \cos \chi_{\mathrm{a}} \\
\dot{Y}_{g}= & V \cos \gamma_{\mathrm{a}} \sin \chi_{\mathrm{a}} \\
\dot{Z}_{g}= & -V \sin \gamma_{\mathrm{a}}
\end{array}\right\}
$$

\subsection{The Change of Mass and Moment of Inertia}

During the motion, the mass of the rocket changes due to the process of discharging combustion products. This change is determined by solving the internal ballistics problem of the rocket motor and can be approximated by the following formula:

$$
m_{d}=|\dot{m}| \cdot t=\frac{m_{\mathrm{P}}}{\tau} t
$$

where

- $m_{d}$ - the amount of mass has been reduced until time $t$,

- $m_{\mathrm{p}}$ - the propellant mass,

- $\dot{m}$ - the velocity of the exhaust process,

- $\tau$ - the propellant burning time.

The mass reduction process leads to a change of the moment of inertia respect to the axes of the rocket; the process is modeled in plane $O X Z$, as shown in Fig. 4.

Suppose the principal moment of inertia respect to the geometrical symmetry axis is $I_{\mathrm{s}}$; equatorial inertia moment is $I_{e}$, then: 


$$
\begin{aligned}
& I_{x}=I_{\mathrm{s}}-m \cdot e_{\mathrm{m}}^{2} \\
& I_{y}=I_{z}=I_{e}
\end{aligned}
$$

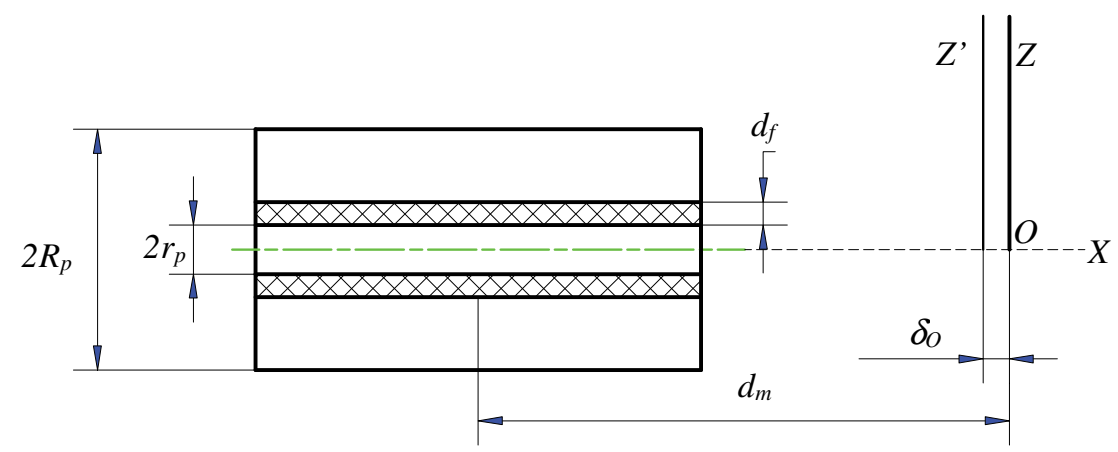

Fig. 4 The change in the rocket's mass

After the interval of time, the change of mass is $\delta m$, the corresponding moment of inertia is:

$$
\begin{aligned}
& I_{x}^{\prime}=I_{x}-\frac{1}{2} \delta m \cdot\left[\left(r_{\mathrm{p}}+d_{f}\right)^{2}-r_{\mathrm{p}}^{2}\right] \\
& I_{y}^{\prime}=I_{z}^{\prime}=I_{z}-\delta m \cdot d_{m}^{2}-(m-\delta m) \cdot \delta_{O}^{2}
\end{aligned}
$$

where

- $R_{\mathrm{p}} ; r_{\mathrm{p}}$ - the outer radius and inner radius of propellant charge, respectively,

- $d_{f}$ - the burning thickness of propellant,

- $d_{m}$ - the distance between mass elements $\delta m$ and axis $O Z$,

- $\delta_{O}$ - the displacement of center of gravity $O$ in the axial direction $O X$.

\subsection{Expressions of Aerodynamic Functions}

The aerodynamic coefficients used for simulating the rocket motion are taken from a rocket of a similar shape in [5], as shown in Tab. 1.

In the table below:

- $C_{A}$ - the total axial force coefficient,

- $C_{N \alpha}$ - the normal force coefficient derivative with the angle of attack (Which corresponds to the projection of the resultant aerodynamic force on the axis $O Z$ ),

- $C_{N \dot{\alpha}}-$ the normal force coefficient derivative with rate of change of the angle of attack,

- $C_{l 0}$ - the total roll moment coefficient,

- $C_{l p}-$ the rolling moment coefficient derivative with roll rate,

- $C_{m \alpha} ; C_{m \dot{\alpha}} ; C_{m q}$ - the pitching moment coefficient derivative with the angle of attack; the rate of change of the angle of attack and pitch rate, respectively.

For aerodynamic coefficients in Tab 1 , the coefficients $C_{A}, C_{l 0}$, and $C_{l p}$ correspond to the angles $\alpha=0$ and $\beta=0$. The remaining coefficients correspond to the angles $\beta=0$ and $\Psi=0$. Because the rocket is axially symmetrical, aerodynamic expressions for the angles $\alpha$ and $\beta ; \Theta$ and $\Psi$ are the same. Then, $C_{N \beta}=C_{N \alpha} ; C_{N \dot{\beta}}=C_{N \dot{\alpha}} ; C_{n \beta}=C_{m \alpha} ; C_{n \dot{\beta}}=C_{m \dot{\alpha}}$; 
$C_{n r}=C_{m q}$ where $C_{N \beta} ; C_{N \dot{\beta}}$ are aerodynamic coefficients corresponding to the projection of the resultant aerodynamic force on the axis $O Y ; C_{n \beta} ; C_{n \dot{\beta}} ; C_{n r}$ are yawing moment coefficient derivative with sideslip angle; the rate of change of sideslip angle and yaw rate, respectively;

Tab. 1 Aerodynamic parameters of the rocket [5]

\begin{tabular}{|c|c|c|c|c|c|c|c|c|}
\hline Mach & $\begin{array}{c}C_{A} \\
{[-]}\end{array}$ & $\begin{array}{c}C_{N \alpha} \\
{\left[\mathrm{rad}^{-1}\right]}\end{array}$ & $\begin{array}{c}C_{N \dot{\alpha}} \\
{[-]}\end{array}$ & $\begin{array}{c}C_{l 0} \\
{[-]}\end{array}$ & $\begin{array}{c}C_{l p} \\
{[-]}\end{array}$ & $\begin{array}{c}C_{m \alpha} \\
{\left[\mathrm{rad}^{-1}\right]}\end{array}$ & $\begin{array}{c}C_{m \dot{\alpha}} \\
{[-]}\end{array}$ & $\begin{array}{c}C_{m q} \\
{[-]}\end{array}$ \\
\hline 0.2 & 0.340 & 8.57 & -52.1 & 0.075 & -8.2 & -53.9 & -96.6 & -2543 \\
\hline 0.4 & 0.305 & 9.10 & -52.1 & 0.076 & -8.3 & -54.4 & -99.4 & -2547 \\
\hline 0.6 & 0.291 & 9.48 & -52.1 & 0.077 & -8.4 & -55.1 & -104.6 & -2539 \\
\hline 0.8 & 0.290 & 9.82 & -52.1 & 0.076 & -8.4 & -54.4 & -112.6 & -2473 \\
\hline 1.0 & 0.391 & 9.99 & -52.6 & 0.077 & -8.4 & -55.0 & -124.0 & -2423 \\
\hline 1.1 & 0.445 & 10.16 & -53.0 & 0.094 & -8.7 & -62.0 & -131.2 & -2676 \\
\hline 1.2 & 0.444 & 10.00 & -53.0 & 0.102 & -8.9 & -65.7 & -140.0 & -2781 \\
\hline 1.3 & 0.349 & 10.08 & -63.5 & 0.110 & -9.0 & -66.0 & -144.5 & -2877 \\
\hline 1.4 & 0.345 & 10.34 & -65.9 & 0.123 & -10.0 & -73.4 & -147.4 & -3068 \\
\hline 1.5 & 0.333 & 10.44 & -67.0 & 0.128 & -10.0 & -70.0 & -148.8 & -2281 \\
\hline 1.6 & 0.322 & 10.59 & -67.7 & 0.126 & -9.8 & -68.0 & -148.8 & -2282 \\
\hline 1.8 & 0.304 & 10.67 & -68.6 & 0.120 & -9.3 & -63.0 & -150.8 & -2175 \\
\hline 2.0 & 0.287 & 10.13 & -68.9 & 0.113 & -8.7 & -57.7 & -151.2 & -2188 \\
\hline 2.2 & 0.271 & 9.05 & -68.7 & 0.105 & -8.1 & -37.7 & -150.9 & -1841 \\
\hline
\end{tabular}

Resultant normal force coefficients corresponding to the angles $\alpha$ and $\beta$ are denoted $C_{N}^{\alpha}$ and $C_{N}^{\beta}$. In accordance with the aerodynamic coefficients in Tab. 1 for rockets, $C_{N}^{\alpha}$ and $C_{N}^{\beta}$ are determined using the following formula [6-8]:

$$
\begin{aligned}
& C_{N}^{\alpha}=C_{N \alpha} \cdot \alpha+\frac{\bar{c}}{2 V} C_{N \dot{\alpha}} \cdot \dot{\alpha} \\
& C_{N}^{\beta}=C_{N \beta} \cdot \beta+\frac{\bar{c}}{2 V} C_{N \dot{\beta}} \cdot \dot{\beta} \\
& C_{N \beta}=C_{N \alpha} ; C_{N \dot{\beta}}=C_{N \dot{\alpha}}
\end{aligned}
$$

where $\bar{c}$ is the rocket diameter.

$C_{A} ; C_{N}^{\alpha} ; C_{N}^{\beta}$ are the aerodynamic coefficients on the Body coordinate system $O X Y Z$. Their conversion to the velocity coordinate system is represented by the conversion matrix between the two coordinate systems as shown below: 


$$
\left[\begin{array}{l}
C_{D} \\
C_{L} \\
C_{Y_{a}}
\end{array}\right]=\left[\begin{array}{ccc}
\cos \alpha \cos \beta & -\cos \alpha \sin \beta & \sin \alpha \\
\sin \beta & \cos \beta & 0 \\
-\sin \alpha \cos \beta & \sin \alpha \sin \beta & \cos \alpha
\end{array}\right]\left[\begin{array}{c}
C_{A} \\
C_{N}^{\alpha} \\
C_{N}^{\beta}
\end{array}\right]
$$

Accordingly, the aerodynamic force and moment components are determined by the formula [6-8]:

$$
\begin{aligned}
& D=\bar{q} \cdot S \cdot C_{D} \\
& L=\bar{q} \cdot S \cdot C_{L}(\alpha) \\
& Y_{\mathrm{a}}=\bar{q} \cdot S \cdot C_{Y_{\mathrm{a}}}(\beta) \\
& L_{R}=\bar{q} S \bar{c}\left(C_{l 0}+\frac{\bar{c}}{2 V} C_{l p} \cdot \omega_{x}\right) \\
& M=\bar{q} S \bar{c}\left(C_{m \alpha} \cdot \alpha+\frac{\bar{c}}{2 V} C_{m \dot{\alpha}} \cdot \dot{\alpha}+\frac{\bar{c}}{2 V} C_{m q} \cdot \omega_{y}\right) \\
& N=\bar{q} S \bar{c}\left(C_{n \beta} \cdot \beta+\frac{\bar{c}}{2 V} C_{n \dot{\beta}} \cdot \dot{\beta}+\frac{\bar{c}}{2 V} C_{n r} \cdot \omega_{z}\right)
\end{aligned}
$$

where $q$ is the dynamic pressure, $\bar{q}=\frac{1}{2} \rho V^{2}$ with $\rho$ is the air density.

\subsection{Motion Trajectory}

The internal and external ballistic parameters of the rocket are taken from [9], and shown in Tabs 2 and 3.

Tab. 2 Internal ballistic parameters of the rocket [9]

\begin{tabular}{|c|l|c|c|}
\hline List & \multicolumn{1}{|c|}{ Quantity } & Value & Unit \\
\hline 1 & Propellant mass & 20.5 & $\mathrm{~kg}$ \\
\hline 2 & Propellant burning time & 1.88 & $\mathrm{~s}$ \\
\hline 3 & Mean thrust & 21000 & $\mathrm{~N}$ \\
\hline
\end{tabular}

Tab. 3 External ballistic parameters of the rocket [9]

\begin{tabular}{|c|l|c|c|}
\hline List & \multicolumn{1}{|c|}{ Quantity } & Value & Unit \\
\hline 1 & Diameter of the rocket & 0.122 & $\mathrm{~m}$ \\
\hline 2 & Mass of the rocket & 66.6 & $\mathrm{~kg}$ \\
\hline 3 & Length of the rocket & 2.870 & $\mathrm{~m}$ \\
\hline 4 & $\begin{array}{l}\text { Distance from the center of gravity to } \\
\text { the bottom of the rocket }\end{array}$ & 1.751 & $\mathrm{~m}$ \\
\hline 5 & $\begin{array}{l}\text { Distance from the center of gravity to } \\
\text { the distributed center of the motor nozzle } \\
\text { system }\end{array}$ & 1.513 & $\mathrm{~m}$ \\
\hline
\end{tabular}




\begin{tabular}{|c|l|c|c|}
\hline List & \multicolumn{1}{|c|}{ Quantity } & Value & Unit \\
\hline 6 & $\begin{array}{l}\text { Moment of inertia respect to the rocket's } \\
\text { symmetry axis }\end{array}$ & 0.149 & $\mathrm{~kg} \cdot \mathrm{m}^{2}$ \\
\hline 7 & Equatorial inertia moment & 51 & $\mathrm{~kg} \cdot \mathrm{m}^{2}$ \\
\hline 8 & $\begin{array}{l}\text { Time the rocket moves on the launcher at } \\
\text { firing angle } 30^{\circ}\end{array}$ & 0.12 & $\mathrm{~s}$ \\
\hline
\end{tabular}

The system of Eq. (16) is solved by the fourth-order Runge-Kutta method with a case of the initial condition as follows [9]:

$+V_{0}=40 \mathrm{~m} / \mathrm{s} ; \gamma_{a 0}=30^{\circ} ; \alpha_{0}=\beta_{0}=\chi_{a 0}=0 ; \dot{\gamma}_{a 0}=-0.09 \mathrm{rad} / \mathrm{s} ; \dot{v}_{0}=0.02 \mathrm{rad} / \mathrm{s}$,

+ The rocket does not contain the mentioned disturbance factors: $e_{\mathrm{T}}=0 ; e_{\mathrm{m}}=0 ; \lambda_{\mathrm{T}}=0$.

Characteristics of the angle of attack and the sideslip angle are shown in Fig. 5; the rocket's trajectory in the firing plane is shown in Fig. 6.

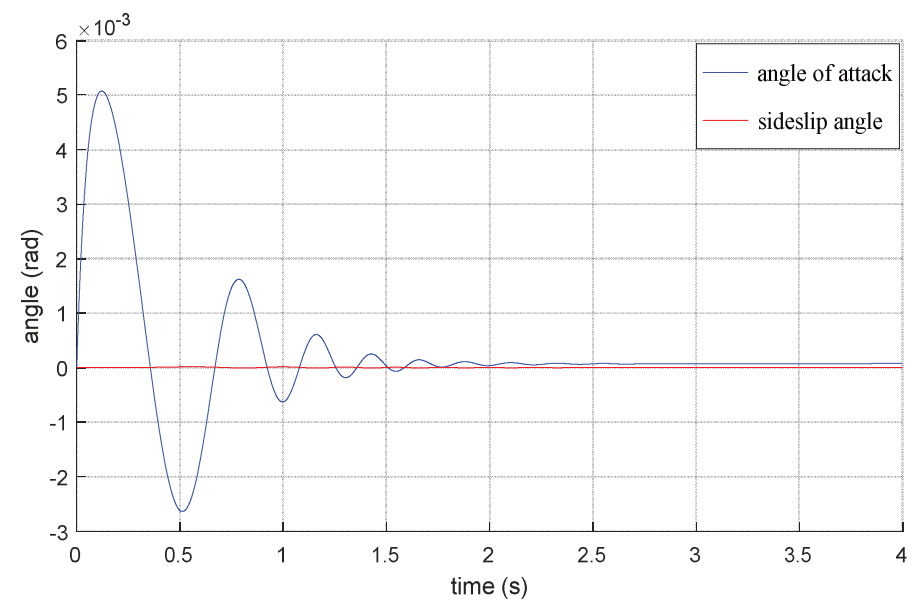

Fig. 5 Characteristics of the angle of attack and the sideslip angle on time

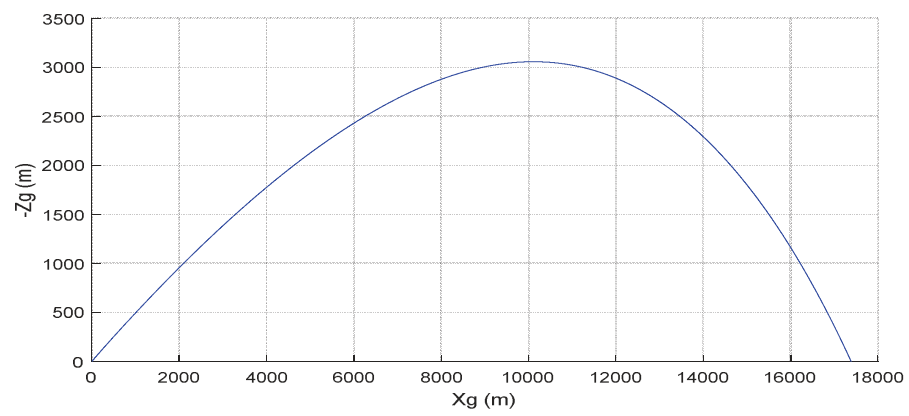

Fig. 6 Trajectory of the rocket in the firing plane

The comparison between the calculated values and the values given in the firing table is shown in Tab. 4. 
Tab. 4 Comparison between the calculated values and the values given in the firing table

\begin{tabular}{|l|c|c|c|c|c|}
\hline \multicolumn{1}{|c|}{ Quantity } & Sign & $\begin{array}{c}\text { Calculated } \\
\text { value }\end{array}$ & $\begin{array}{c}\text { Value in firing } \\
\text { table }\end{array}$ & Deviation & Unit \\
\hline Firing range & $X_{\max }$ & 17387 & 17000 & $+2.27 \%$ & $\mathrm{~m}$ \\
\hline Altitude & $-Z_{\max }$ & 3058 & 2900 & $+5.45 \%$ & $\mathrm{~m}$ \\
\hline Lateral deviation & $Y_{\max }$ & -0.7 & $(-130 ; 130)$ & & $\mathrm{m}$ \\
\hline Flight time & $t_{\max }$ & 50.1 & 48 & $+4.37 \%$ & $\mathrm{~s}$ \\
\hline
\end{tabular}

Fig. 5 shows that when the amplitude of attack angle decreases to zero and the sideslip angle is almost zero, the rocket motion on the flight is stable. The amplitude of the angle of attack is greater due to the influence of gravity which generates the initial angular velocity for the rocket axis in the vertical plane. Trajectory elements from calculating agree well with the corresponding data in the firing table. Therefore, the mathematical model and the aerodynamic coefficient ensure the required accuracy.

\section{Effect of Thrust Deflection and the Deviation of Center of Gravity on Mo- tion Stability of Fin-Stabilized Rocket}

The influence of the thrust deflection and deviation of the center of gravity on the stability of the rocket motion are evaluated by comparing the trajectories containing the disturbance factors and the standard trajectory which does not contain disturbances. Some parameters are compared including the characteristic of the angle of attack and the characteristic of the sideslip angle and deviation of the trajectory falling points.

The characteristics of the angle of attack and the sideslip angle demonstrate the rocket's ability to maintain the standard direction in space, so they represent the level of the rocket's stability on the flight; the deviation of the falling point represents the accuracy of the trajectory compared to the standard trajectory. The rocket is stable if both above-mentioned requirements are met.

The trajectory used for the investigation is presented in section 3.2. According to [10] and [11], the allowable deviation of the falling point corresponds to the range of $X_{\max }=17387 \mathrm{~m}$ as: $\Delta X=428 \mathrm{~m} ; \Delta Y=536 \mathrm{~m}$.

Some cases of investigating the motion stability of the rocket in the presence of the mentioned disturbance factors are presented in the following sections.

\subsection{There is Only Thrust Deflection}

When considering only the presence of the thrust deflection, then $e_{\mathrm{m}}=0 ; \varepsilon_{\mathrm{m}}=0$; A few cases of the thrust deflection were surveyed as:

a) $\varepsilon_{\Gamma}=0 ; \lambda_{\mathrm{T}} \neq 0$

The mathematical model is solved with different values of $\lambda_{\mathrm{T}}$ and then the trajectory deviations are compared with the allowable deviations. The maximum value of the thrust deflection angle obtained was $\lambda_{\mathrm{T}}=0.002 \mathrm{rad}$. The stability characteristics of the trajectory are investigated corresponding to the values: $\lambda_{\mathrm{T}}=0.0005 ; 0.001 ; 0.0015 ; 0.002 \mathrm{rad}$. The characteristics of the angle of attack, the sideslip angle and deviation of the falling point (compared to the standard trajectory) are shown in Figs 7-9. 


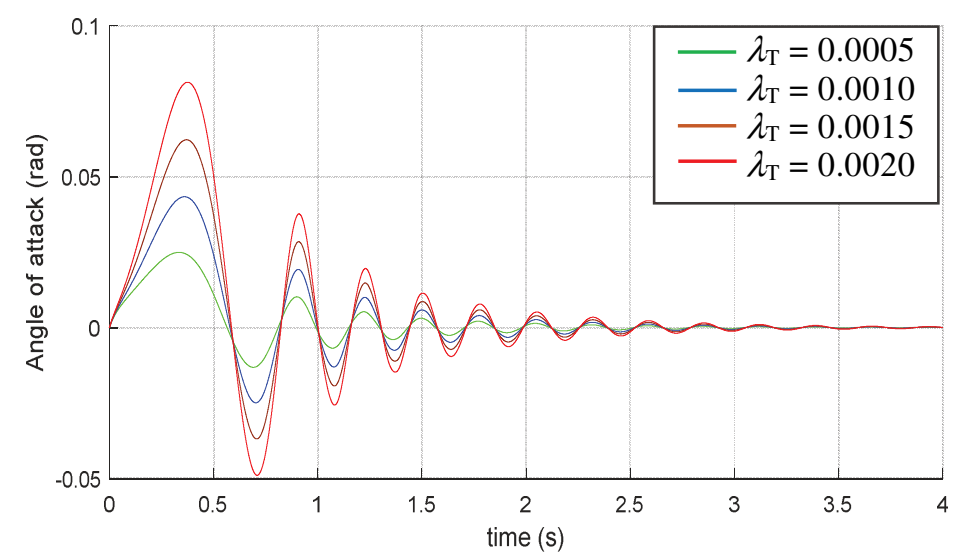

Fig. 7 Dependence of angle of attack on time

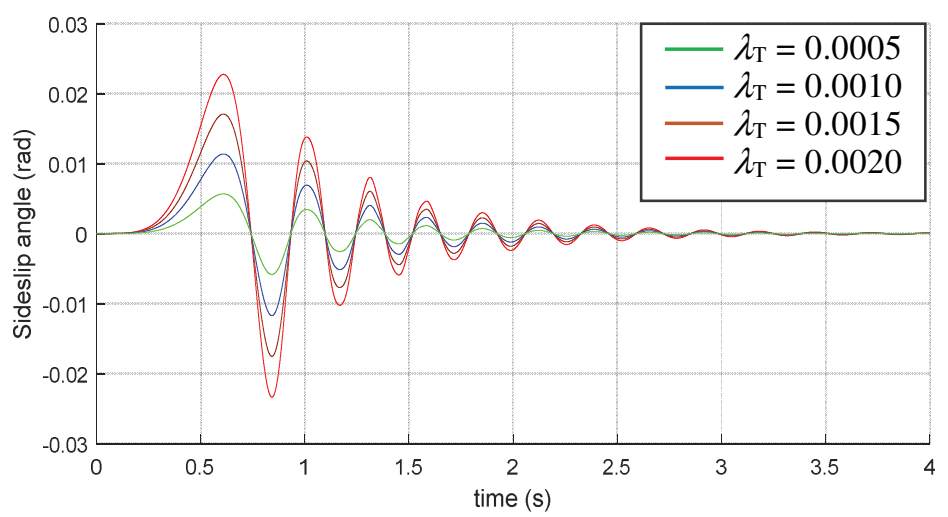

Fig. 8 Dependence of sideslip angle on time

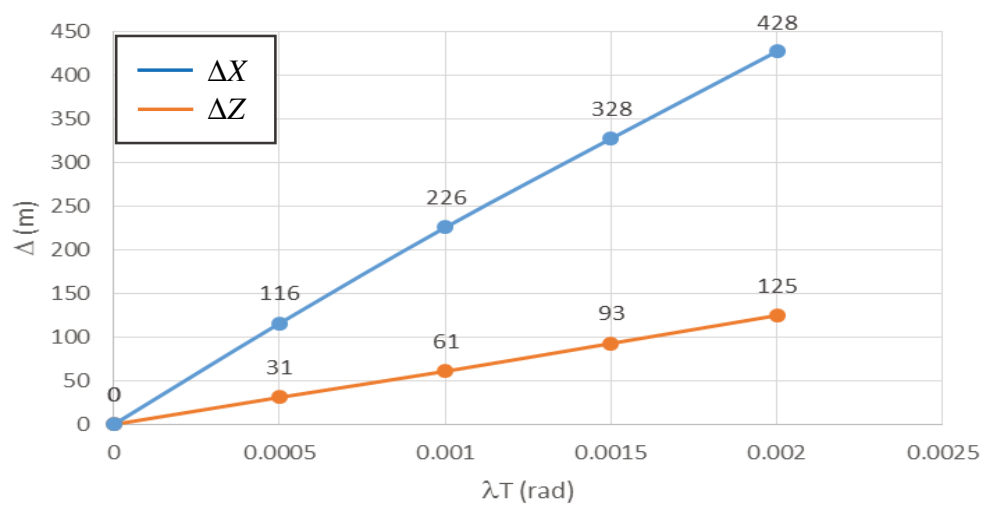

Fig. 9 Dependence of the falling point deviation on the thrust deflection angle 


\section{Discussion}

According to Figs 7-9, some comments are drawn as follows:

- the oscillations of the angle of attack and sideslip angle attenuate to zero so the rocket oscillations on the flight are stable with these given angles. When $\lambda_{\mathrm{T}}$ increases, the amplitude of the angle of attack and sideslip angle also increases while frequency and turned-off time is the same, so instability increases,

- for each given value of $\lambda_{\mathrm{T}}$, the amplitude of the angle of attack is greater than the amplitude of sideslip angle. This can be explained as follows: at the initial time, the thrust is in the vertical plane, the moment in the vertical plane is great but the moment in the horizontal plane is zero. In the next short period of time, due to the small rocket's spin motion is, the moment formed in the horizontal plane increases slowly while the moment in the vertical plane decreases slowly. In addition, the influence of gravity also results in a faster increase in the amplitude of the angle,

- several other computational results for trajectories with thrust deflections that are slightly greater than the allowable value $\left(\lambda_{\mathrm{T}}=0.002 \mathrm{rad}\right)$ show that the rocket motions on their flight are also stable. Based on this, the conclusion can be drawn that when only taking into account the mentioned disturbance factors, if trajectories ensure the allowable deviation of the falling point, the rocket motions on the flight are stable but the opposite is not surely true,

- the deviation of falling point increases and is linearly dependent on the increase of the thrust deflection. Because in the motion equation Eq. (16), parameter $\lambda_{\mathrm{T}}$ is expressed in function $\sin \lambda_{\mathrm{T}}$, the change of the trajectory elements at each time depends on the change of function $\sin \lambda_{\mathrm{T}}$, when $\lambda_{\mathrm{T}}$ is very small, $\sin \lambda_{\mathrm{T}} \approx \lambda_{\mathrm{T}}$, so this dependency is almost linear. The deviation of the falling point in range direction increases faster than the deviation in the azimuth direction which is due to the amplitude of the angle of attack that is greater than the angular amplitude.

b) $\lambda_{\mathrm{T}}=0.0015 \mathrm{rad} ; \varepsilon_{\mathrm{T}}=0^{\circ} ; 30^{\circ} ; 6^{\circ} ; \mathbf{9 0}^{\circ}$

The characteristics of the angle of attack; the sideslip angle and deviation of the falling point are shown in Figs 10-12.

\section{Discussion}

According to Figs 10-12, some comments are drawn as follows:

- the rocket motions on the flight are stable. When the angle $\varepsilon_{\mathrm{T}}$ increases from $0^{\circ}$ to $90^{\circ}$, the amplitudes of the angle of attack decrease, but the amplitudes of the sideslip angle increase. That is due to the decrease of the moment arising in the vertical plane and the increase of the moment arising in the horizontal plane,

- the dependence of the falling point deviation on $\varepsilon_{\mathrm{T}}$ is sinusoidal. That is due to the term $\sin \varepsilon_{\mathrm{T}}$ in Eq. (16), when the angle $\varepsilon_{\mathrm{T}}$ is large, the change of trajectory elements at each time depends on the change of $\sin \varepsilon_{\mathrm{T}}$,

- in Fig. 12, in the interval, $56^{\circ} \leq \varepsilon_{\mathrm{T}} \leq 102^{\circ}$, the deviations of the falling point in the azimuth direction exceed the allowed value. Meanwhile, the oscillations corresponding to angles $56^{\circ} \leq \varepsilon_{\mathrm{T}} \leq 90^{\circ}$ are stable on the flight. This result indicates that although the oscillations are stable on the flight path, the firing accuracy may not be guaranteed,

- the deviation graphs get extremums at the angles $\varepsilon_{\mathrm{T}}$ approximately equal to $0^{\circ}$ and $90^{\circ}$. 


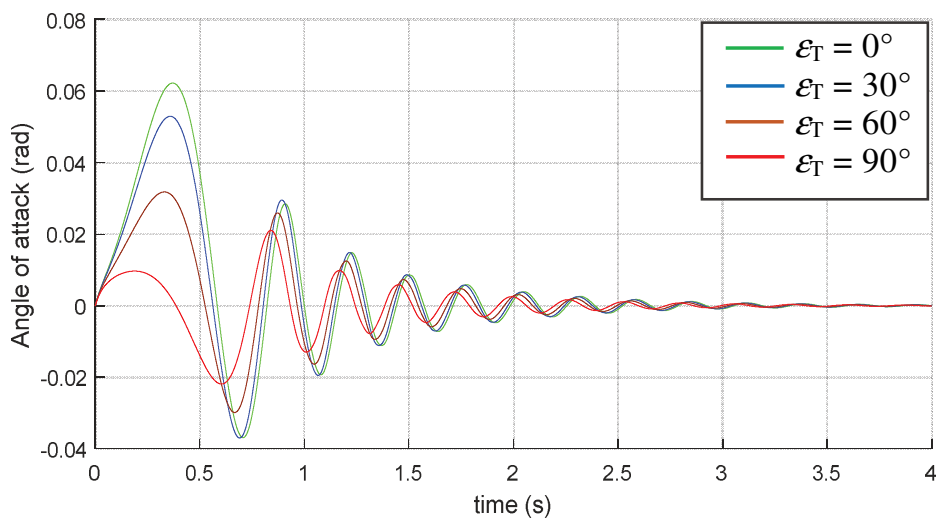

Fig. 10 Dependence of angle of attack on time

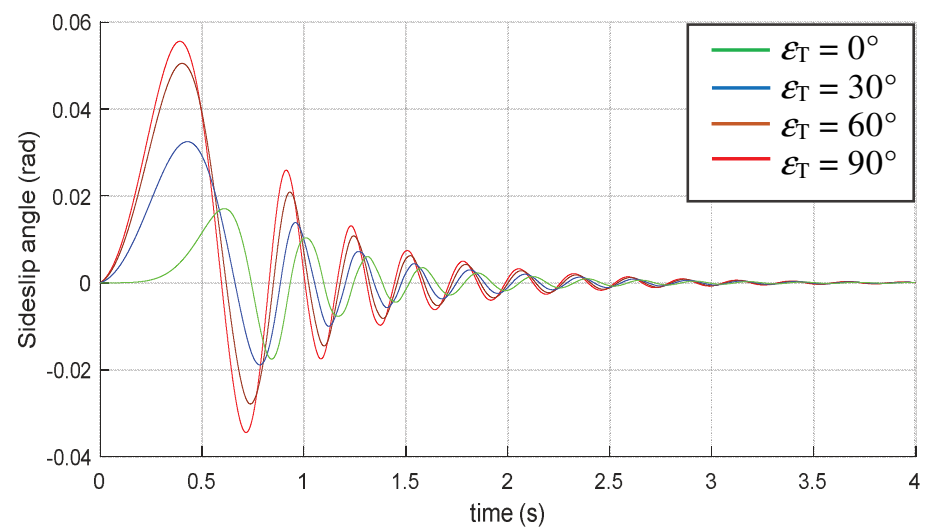

Fig. 11 Dependence of sideslip angle on time

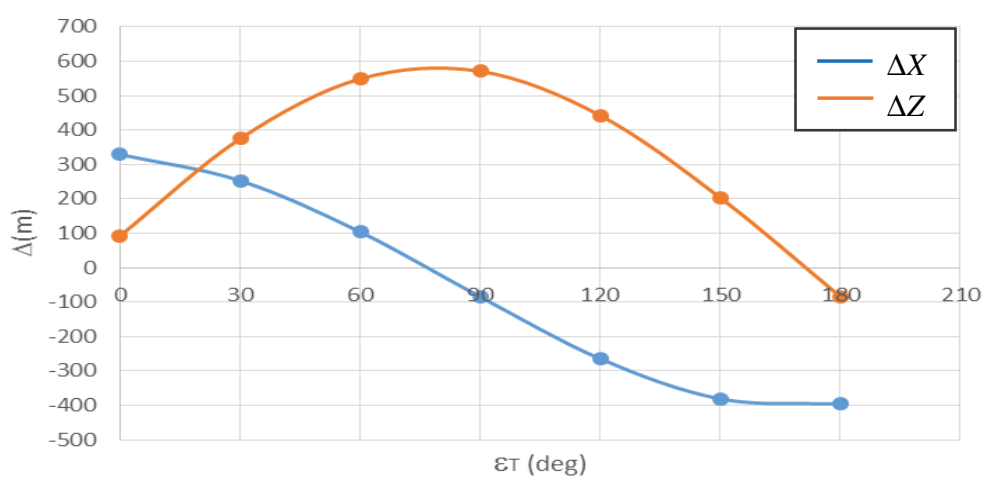

Fig. 12 Dependence of the falling point deviation on the thrust deflection angle 


\subsection{Deviation of the Center of Gravity}

When considering only the presence of deviation of the center of gravity, then $\lambda_{\mathrm{T}}=0$; $\varepsilon_{\mathrm{T}}=0$.

Consider the case of deviation of the center of gravity in the vertical plane, $\varepsilon_{\mathrm{m}}=0$. The mathematical model is solved and then the trajectory deviations are compared with the allowable values. The maximum value of deviation of the center of gravity $e_{\mathrm{m}}$ is $0.0024 \mathrm{~m}$. The stability characteristics of the trajectory are investigated for the values: $e_{\mathrm{m}}=(0.0005 ; 0.001 ; 0.0017 ; 0.0024) \mathrm{m}$. Deviations of the falling point (compared to the standard trajectory) are shown in Fig. 13.

\section{Discussion}

This case is similar to the case of $4.1-\mathrm{a}$, but the moments generated in the planes are opposite to the case of 4.1-a, so the characteristics of the angle of attack, sideslip angle and deviations of falling point are in the opposite direction to case of 4.1-a.

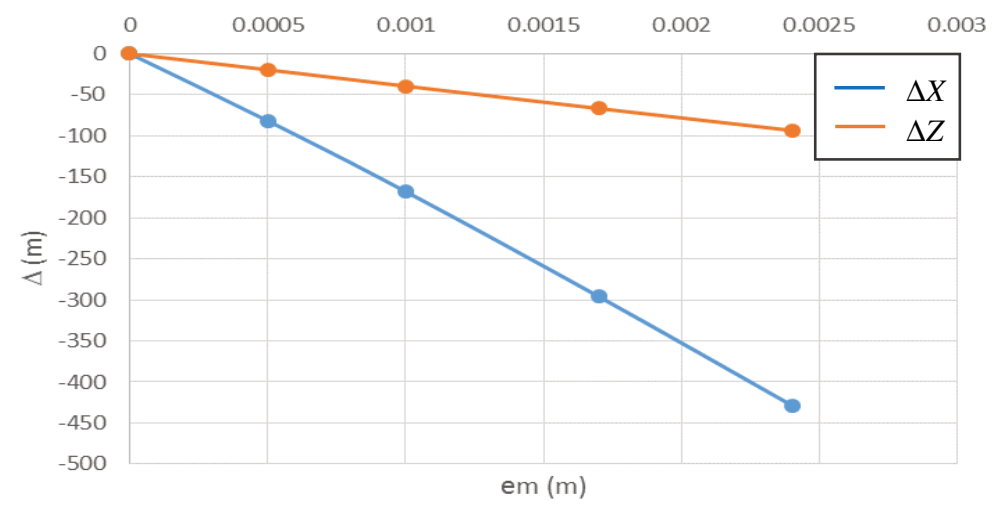

Fig. 13 Dependence of the falling point deviation on the deviation of the center of gravity

\subsection{Combination of Thrust Deflection and Deviation of Center of Gravity}

According to the calculation results presented above, when not taking into account the initial disturbance factors and appearing on the flight, then the stability of the rocket motion can be assessed by the deviation of the falling point. Also in actual combat, the accuracy of the falling point is one of the most important criteria. These reasons show that it is necessary to determine the limits of disturbance factors for ensuring the firing accuracy. Some calculation results that determine the limits of mentioned disturbance factors when combining them are presented in Tab. 5:

Tab. 5 Some limits of disturbance factors

\begin{tabular}{|c|c|c|c|}
\hline$\varepsilon_{\mathrm{T}}\left[{ }^{\circ}\right]$ & $\varepsilon_{\mathrm{m}}\left[{ }^{\circ}\right]$ & $\lambda_{\mathrm{T}}[\mathrm{rad}]$ & $e_{\mathrm{m}}[\mathrm{m}]$ \\
\hline 0 & 0 & 0.0015 & $(-0.0008,0.00464)$ \\
\hline 0 & 0 & 0.0010 & $(-0.00153,0.00388)$ \\
\hline 0 & 60 & 0.0010 & $(-0.0024,0.00233)$ \\
\hline 90 & 60 & 0.0010 & $(-0.00444,0.00077)$ \\
\hline
\end{tabular}




\section{Discussion}

The results in Tab. 5 show that, for each disturbance factor, there is a set of values for the remaining disturbance factors, respectively, determining their limits, ensuring the accuracy of the trajectory. The limits are different. Based on the limits, it can be determined whether the rocket motion is stable or not.

\section{Conclusion}

The article established a mathematical model for unguided-rockets motion taking into account the thrust deflection and the deviation of the center of gravity. The mathematical model is solved for a specific unguided-rocket to predict its standard trajectory and deviation trajectories, thereby evaluating the effect of thrust deflection and deviation of the center of gravity on the rocket's motion stability.

All mentioned turbulence factors cause instability to rockets on the flight and cause the falling point deviation. The deviation of the falling points is almost linearly dependent on $\lambda_{\mathrm{T}}$ and $e_{\mathrm{m}}$ and sinusoidal dependent on $\varepsilon_{\mathrm{T}}$ and $\varepsilon_{\mathrm{m}}$. In addition, disturbance factors affect each other, increasing or reducing the influence of the remaining factors on the rocket's motion stability. When considering only the mentioned disturbance factors, the falling point accuracy can be considered as a criterion to evaluate rocket's motion stability. The determination of limits for disturbance factors when they combine together is important in the exploitation and use of rockets containing disturbances.

The results in this article can be a reference for the calculating, designing and using rockets.

\section{References}

[1] LE, A.D., NGUYEN, H.D. and TRAN, L.N. Flight Theory and Air Defense Missile Control System (in Vietnamese). Hanoi: Military Technical Academy, 1998. 166 p.

[2] NGUYEN, V.T. and NGUYEN, D.S. External Ballistic Textbook (in Vietnamese). Hanoi: Military Technical Academy, 2003. 379 p.

[3] KONEČNÝ, P. Exterior Ballistics of Rockets. Brno: University of Defence in Brno, 2006. 128 p. ISBN 978-80-7231-200-9.

[4] MORARU, F. and SAFTA, D. Mathematical Model for the Rocket General Motion Simulation in Presence of Various Perturbations. In Proceedings of the $5^{\text {th }}$ Conference on Weapon Systems. Brno: Military Academy, 2001, p. 66-81.

[5] KHALIL, M., ABDALlA, H. and KAMAL, O. Trajectory Prediction for a Typical Fin Stabilized Artillery Rocket. In Proceedings of the $13^{\text {th }}$ International Conference on Aerospace Sciences \& Aviation Technology. Cairo: Military Technical College, 2009, p. 1-14. DOI 10.21608/asat.2009.23742.

[6] CAUGHEY, D. Introduction to Aircraft Stability and Control [online]. New York: Sibley School of Mechanical \& Aerospace Engineering, 2011. 153 p. [viewed 201911-20]. Available from: https://courses.cit.cornell.edu/mae5070/Caughey_2011_04.pdf

[7] GRAUER, J., MORELLIY, E. and MURRIZ, D. Flight Test Techniques for Quantifying Pitch Rate and Angle of Attack Rate Dependencies. Journal of Aircraft, 2017, vol. 54, no. 6, p. 2367-2377. DOI 10.2514/1.C034407.

[8] CHARUBHUN, W., CHUSILP, P. and NUTKUMHANG, N. Effects of Aerodynamic Coefficient Uncertainties on Trajectory Simulation of a Short-range Solid 
Propellant Free Rocket [online]. In Proceedings of the $26^{\text {th }}$ International Symposium on Ballistics. Miami, 2011. [viewed 2019-11-12]. Available from: https://www.academia.edu/7623368/EFFECTS_OF_AERODYNAMIC_COEFFICIENT _UNCERTAINTIES_ON_TRAJECTORY_SIMULATION_OF_A_SHORT-RANGE_ SOLID_PROPELLANT_FREE_ROCKET

[9] NGUYEN, D.P. Researching the Effects of Some Initial Disturbance Factors on Firing Accuracy (in Vietnamese) [PhD Thesis]. Hanoi: Military Technical Academy, 2016. $134 \mathrm{p}$.

[10] Jet Bullets M21-OФ-VN. Industry standards 06TCN743 (in Vietnamese). Vietnam, 1999.

[11] Fighting Vehicle BM-21 (in Russian) [Technical Specifications]. Moscow: Military Publishing House, 1980. 99 p. 